\section{P595 VAGINAL MICROBIOTA AMONG ADOLESCENT AND YOUNG ADULT WOMEN WITH PELVIC INFLAMMATORY DISEASE}

${ }^{1}$ Maria Trent* ${ }^{*}{ }^{2}$ Jamie Perin, ${ }^{2}$ Pamela Matson, ${ }^{3}$ Charlotte Gaydos. ${ }^{1}$ Johns Hopkins University School of Medicine, Ped Gen Pediatrics Adoles Medicine, Baltimore, USA; ${ }^{2} J o h n s$ Hopkins School of Medicine, Pediatrics, Baltimore, USA; ${ }^{3}$ Johns Hopkins University, Division of Infectious Diseases, Baltimore, USA

\subsection{6/sextrans-2019-sti.665}

Background Pelvic Inflammatory Disease (PID) is a polymicrobial infection currently treated using syndromic management with broad-spectrum antibiotics. There are limited data describing the vaginal microbiota among adolescent and young adult women with PID, and how the post-PID microbial state may predispose to subsequent infection due to ongoing infection and shifts in vaginal microbiota. The purpose of this pilot research is to examine the microbial environment among adolescent and young adult women with acute PID.

Methods This analysis utilizes stored samples from 13-25-yearold patients $(n=26)$ diagnosed with acute PID and enrolled in the Technology Enhanced Community Health Nursing (TECHN) study, a large randomized controlled clinical trial designed to test a multi-faceted intervention for prevention of PID. Vaginal microbiota was characterized by $16 \mathrm{~S}$ rRNA gene sequencing (V3-V4 regions) and clustered into community state types (CSTs).

Results At baseline, the majority of patients with acute PID were in a low-Lactobacillus or L.iners dominated state (CST I (L. crispatus dominated $(\mathrm{N}=3,11.54 \%)$, CST III L. inersdominated ( $\mathrm{N}=7,26.9 \%)$, CST IV Low-Lactobacillus ( $\mathrm{N}=15$, $57.69 \%)$, CST V L. jensenii-dominated $(\mathrm{N}=1,3.85 \%))$. The single CST V case had a relatively low abundance $(55 \%)$ of L. jensenii.

Conclusion Preliminary vaginal microbiota testing among AYA with PID revealed over $1 / 2$ of participants had a low abundance of Lactobacillus spp indicative of bacterial vaginosis and risk to STI. Over $1 / 4$ had L. iners-dominated microbiotas, which are also often associated with BV. Lactobacillus spp and are thought to protect against pathogens; however, the level of protection may vary by strain. Additional research should examine these findings in larger samples, include PID-negative patients for comparison, and assess the changes in the vaginal microbiota associated with successful clearance of pathogens. Such work may improve understanding of the vaginal microenvironment during PID and elucidate a path to shift from syndromic management to precision treatment among affected patients.

Disclosure No significant relationships.

\section{P597 COMPARISON OF ASSAYS AND SPECIMEN TYPES FOR THE DIAGNOSIS OF MYCOPLASMA GENITALIUM AND MACROLIDE RESISTANT MUTATIONS}

${ }^{1}$ Max Chernesky*, 'Dan Jang, ${ }^{2}$ Irene Martin, 'Anika Shah, 'Marek Smieja, 'David Speicher, ${ }^{1}$ Avery Clavio, ${ }^{1}$ Manuel Arias. 'McMaster University/St. Joseph's Research Institute, Hamilton, Canada; ${ }^{2}$ National Microbiology Laboratory, Winnipeg, Canada

\subsection{6/sextrans-2019-sti.666}

Background The Aptima MG assay (Hologic Inc.) detects Mycoplasma genitalium (MG). SpeeDx Resistance Plus MG (SpeeDx Pty Ltd) simultaneously determines whether the MG detected is a wild-type (WT) or has any of 5 macrolide resistant mutations (MRM) in the 23S rRNA gene. The STD6 ACE assay (Seegene Canada Inc.) which is Health Canada approved and Aptima require further testing for MRM by $23 \mathrm{~S}$ rRNA sequencing. The objectives were to enroll 300 women to self-collect 3 vaginal swabs (VS) and first-void urine (FVU) to be tested for MG in the 3 assays and for MRM.

Methods Aptima was performed on a Panther instrument. STD6 ACE used EasyMag extraction and gel electrophoresis. SpeeDx was extracted on an m2000sp and amplification was on an ABi 7500. Positives from Aptima and STD6 were tested for MRM by sequence typing. Extra positives by Aptima were blindly tested with negatives by alternate assays, detecting distinctly different $16 \mathrm{~S}$ rRNA and $23 \mathrm{~S}$ rRNA. Agreements and Kappa values were calculated for MG detection and MRM.

Results For 190 women, MG-positives by SpeeDX were 24 VS and 17 FVU, compared to 17 VS and 9 FVU by STD6. Aptima detected 34 VS and 29 FVU as positive. Overall agreements and Kappa values were very good K 0.82 (VS) to good K 0.69 (FVU) between Aptima and SpeeDx, but moderate to fair for Aptima or SpeeDx with STD6. Confirmatory testing of extra positives confirmed 79\% (11/14) as positive for Aptima and 0\% (0/6) for STD6. Patient MRM rates from each assay were 60\% (15/25) SpeeDx, 63.6\% (7/11) Aptima and $57.9 \%(11 / 19)$ STD6.

Conclusion Aptima was more sensitive on both VS and FVU. VS was a better sample than FVU for SpeeDx. STD6 lacked sensitivity and specificity for the identification of MG.MRM rates were similar for the three identification and typing systems.

Disclosure No significant relationships.

\section{P598 DETECTION OF CHLAMYDIA, GONORRHOEA AND MYCOPLASMA GENITALIUM IN SEMEN AND IN ESWAB MEDIUM USING HOLOGIC APTIMA ASSAYS}

Chloe Le Roy, Sabine Pereyre, Angelique Alonso-Marro, Bertille De Barbeyrac Cécile Bébéar*. University of Bordeaux, Usc Ea 3671, Bordeaux, France

\subsection{6/sextrans-2019-sti.667}

Background The objectives of this study were to determine the limits of detection (LODs) of Chlamydia trachomatis (CT), Neisseria gonorrhoeae (NG) and Mycoplasma genitalium (MG), using Aptima Combo 2 for CT/NG and Aptima Mycoplasma genitalium assays in presence of different volumes of semen and ESwab medium (bioMérieux, France) used for specimen collection. Furthermore, the preservation conditions of these bacteria in ESwab medium were assessed.

Methods Semen specimens were collected anonymously from patients consulting at the reproductive biology department of Bordeaux University Hospital. To determine LOD, quantified cultures of each bacterium were spiked in semen or ESwab medium. Different volumes added to APTIMA $^{\text {Oे }}$ Specimen transfer tubes were tested with Aptima Combo $2 \mathrm{CT} / \mathrm{NG}$ and Aptima Mycoplasma genitalium assays. Storage conditions (5 days at room temperature and 7 days at $4^{\circ} \mathrm{C}$ ) of each bacterium in ESwab medium were evaluated. All the experiments were performed in triplicate on the Hologic ${ }^{\mathrm{O}}$ Panther system. Results The semen was a slight source of amplification inhibition for CT and MG. However, using a volume of $50 \mu \mathrm{L}$ of semen, the LOD of CT, NG and MG in semen remained low, at $10^{3} \mathrm{IFU} / \mathrm{mL}, 10 \mathrm{CFU} / \mathrm{mL}$ and $10^{2} \mathrm{CCU} / \mathrm{mL}$, respectively. A 